\section{Discussion: Automatic scanning for cervical smears}

\author{
I. M. P. DAWSON
}

We have just heard two very interesting papers on the possibilities of automation in the screening of cervical smears and on the identification of abnormalities in chromosomal karyotypes. My own experience lies in the field of automated cervical cytology where we have developed techniques related to, but distinct from, those which Dr Spriggs has put before you: I have no direct experience in Dr Rutovitz's ${ }^{1}$ field.

This section of the meeting covers automation in histopathology and cytology in which our traditional instrument is the microscope. First, we must differentiate clearly between mechanization and automation, which are not synonymous. Anyone setting out on the thorny pathway of automation as a replacement for microscopic visual assessment must ask himself certain questions. What am I trying to do? Or, put another way, Do I want partial automation in that a machine (I use the term 'machine' in an all-embracing sense) will select fields or cells which I shall then study microscopically or do I want full automation in which the machine classifies the cells present? In other words, who makes the ultimate decision on abnormality-the machine or I?

If I opt for partial automation then material must be presented to the machine in a form in which I can subsequently examine it microscopically: this may severely limit the mechanical measurements which the machine can make. As a simple example, it will probably be impossible to measure cell volume and many automated scanners of the 'flying spot' type do not work too well with blue nuclear stains, but against this one can set the probability that only one or two parameters, such

${ }^{1} \mathrm{Dr}$. Rutovitz's paper is not published but readers may consult his other paper in the British Medical Bulletin, 'Computing in Medicine' Brit. Med. Bull., 1968, 24, 260. as the longest chord of nucleus, will have to be measured.

This leads naturally to the next question, on which Spriggs has already touched, 'If I want full automation what am I going to program my machine to read?' Alternatively, 'Are there differences between a normal and an abnormal cell, and between different patterns of abnormality which a machine can recognize?' We are fond, in discussing the differentiation of, say, malignant squames from inflammatory, of talking about 'subjective impressions' by which we mean the visual comparison of a particular cell with a memory impression of previous cells whose nature we know. My own feeling, with which I know many disagree, is that one's 'subjective impressions' are based on simultaneous assessment of a large number of parameters, such as nuclear size, density of staining, nuclear longest chord, nuclear-cytoplasmic ratio, etc, each of which is capable, at any rate theoretically, of being measured by a machine. Automated recognition of cells should therefore be possible provided that one uses a sufficiently wide multiparameter approach, a view which, if I have understood his paper rightly, Dr Rutovitz would share, at any rate for chromosomes. But if we do this, we must rid ourselves of the idea that the parameters must necessarily all be of visual type or that the method of presentation of material to the automated scanners must necessarily be in a form which can also be used for visual assessment. This many cytologists find hard to accept-but you cannot have it both ways!

If the proposition of full automation is accepted one final question remains, 'Is it worth it?' This has to be argued not only in terms of money, because no one will buy a machine costing $£ 1,000,000$ to do the work of two technicians, but also in terms of time; no one will buy a machine which takes a week to scan a smear. However, it has also to be argued in terms of interest. The first two can probably be overcome but the third, though I am an advocate of total automation in cervical cytology, still disturbs me. I cannot help being reminded of two lines in T. S. Eliot's 'The Rock'

'Where is the wisdom we have lost in knowledge?
Where is the knowledge we have lost in information?' 